\title{
Spectral classification of the mass donors in the high-mass X-ray binaries EXO 1722-363 and OAO 1657-415^
}

\author{
A. B. Mason ${ }^{1}$, J. S. Clark ${ }^{1}$, A. J. Norton ${ }^{1}$, I. Negueruela ${ }^{2}$, and P. Roche $e^{1,3,4}$ \\ 1 Department of Physics \& Astronomy, The Open University, Milton Keynes MK7 6AA, UK \\ e-mail: a.mason@open.ac.uk \\ 2 Departamento de Física, Ingeniería de Sistemas y Teoría de la Señal, Universidad de Alicante, Apdo. 99, 03080 Alicante, Spain \\ 3 School of Physics \& Astronomy, Cardiff University, The Parade, Cardiff, CF24 3AA, UK \\ 4 Division of Earth, Space \& Environment, University of Glamorgan, Pontypridd, CF37 1DL, UK
}

Received 13 May 2009 / Accepted 9 July 2009

\section{ABSTRACT}

\begin{abstract}
Aims. We report near-infrared (NIR) observations of the mass donors of the eclipsing high-mass X-ray binary (HMXB) systems EXO 1722-363 and OAO 1657-415 in order to derive their accurate spectral classifications.

Methods. ESO/VLT observations of the targets with the NIR spectrometer ISAAC were compared with several published NIR spectral atlases of $\mathrm{O}$ and $\mathrm{B}$ supergiants, an identification of each object's spectral characteristics was made, enabling the refinement of spectral classification of the mass donors.

Results. We determined that EXO 1722-363 was of spectral type B0-B1Ia, positioned at a distance $8.0_{-2.0}^{+2.5} \mathrm{kpc}$ with a progenitor mass in the range 30-40 $M_{\odot}$. Luminosity calculations imply that $L_{\mathrm{X}} \sim 10^{35}-10^{37} \mathrm{erg} \mathrm{s}^{-1}$ for this distance range. We conclude that EXO 1722-363 shares many of the properties associated with other X-ray binary B-type supergiant donors.

We found that OAO 1657-415 correlates closely with the spectra of a class of transitional objects, the Ofpe/WNL stars, an intermediate evolutionary stage between massive $O$ type stars leaving the main sequence and evolving into Wolf-Rayets. Due to the wide range in luminosity displayed by Ofpe/WNL stars, $\left(\log \left(L / L_{\odot}\right) \sim 5.3-6.2\right)$ distance determinations are problematic. For OAO 1657-415 we report a distance of $4.4 \leq d \leq 12 \mathrm{kpc}$, implying an X-ray luminosity of $1.5 \times 10^{36} \leq L_{\mathrm{X}} \leq 10^{37} \mathrm{erg} \mathrm{s}^{-1}$. We have used our new classification of OAO 1657-415 to explain the physical processes responsible for its unique position within the Corbet diagram. Ofpe/WNL stars demonstrate a high rate of mass-loss through a dense stellar wind combined with a low terminal velocity. This combination of wind properties leads to a high accretion rate and transfer of angular momentum to the neutron star in this system. We believe this in turn leads to a smaller instantaneous equilibrium spin period with respect to normal OB supergiants.
\end{abstract}

Key words. binaries: eclipsing - binaries: general - X-rays: binaries - stars: winds, outflows - stars: individual: OAO 1657-415 stars: individual: EXO 1722-363

\section{Introduction}

High-mass X-ray binaries (HMXBs) are comprised of massive $\left(\geq 10 M_{\odot}\right.$ ) donor stars and an accreting compact object (a neutron star or black hole). They are typically divided into two main classes, Be/X-ray binaries $(\mathrm{Be} / \mathrm{X})$ and Supergiant X-ray binaries (SGXRBs). Be/X binaries form the majority $(\sim 60 \%)$ of HMXB systems (Liu et al. 2006), consisting of a neutron star which accretes matter from the circumstellar equatorial disc of a Be star. These systems have wide orbits and moderate eccentricities, exhibiting two main types of transient X-ray outbursts. Type I occur at the periastron passage of the neutron star with $L_{X} \sim 10^{36}-10^{37} \mathrm{erg} \mathrm{s}^{-1}$. Type II outbursts, which are not correlated with the orbital period, display luminosities of $L_{X} \geq 10^{37} \mathrm{erg} \mathrm{s}^{-1}$ (Okazaki \& Negueruela 2001). SGXRBs have counterparts which are early type supergiants and accrete from either Roche-lobe overflow or a radially outflowing stellar wind. They are persistent sources of X-ray emission, with stellar wind fed systems having a lower flux than $\mathrm{Be} / \mathrm{X}$ systems $\left(L_{\mathrm{X}} \sim 10^{35}\right.$ $\left.10^{36} \mathrm{erg} \mathrm{s}^{-1}\right)$. For stars that fill their Roche lobe a much higher $\mathrm{X}$-ray luminosity can be achieved of $\sim 10^{38} \mathrm{erg} \mathrm{s}^{-1}$ (Liu et al. 2006).

* Based on observations carried out at the European Southern Observatory under programme ID 081.D-0073(A).
The Corbet diagram (Corbet 1986) describes the relationship between orbital period $P_{\text {orb }}$ and pulse period $P_{\mathrm{s}}$ for an accretion powered pulsar in a HMXB system. The correlation $P_{\mathrm{s}} \propto P_{\mathrm{orb}}^{4 / 7}$ for wind fed systems was found, contrasting with that for $\mathrm{Be} / \mathrm{X}$ systems of $P_{\mathrm{s}} \propto P_{\mathrm{orb}}^{2}$. Each seperate class lies in a distinct location on the Corbet diagram allowing a differentiation to be made between $\mathrm{Be} / \mathrm{X}$ and both underfilled and filled Roche-lobe SGXRBs systems.

Eclipsing X-ray pulsar systems provide a means to accurately determine the mass of the neutron star. Such systems are of significant importance, as they are the only binary accreting system in which the neutron star mass may be measured, providing insights and constraints on the neutron star equationof-state. Unfortunately, only ten eclipsing HMXB systems have been identified within the Galaxy, meaning that the characterisation of further examples is a priority. In this paper we undertake the first step in this process for two systems in which the neutron star masses have yet to be measured; the classification of the mass donors in EXO 1722-363 and OAO 1657-415.

\subsection{EXO 1722-363}

EXO 1722-363 (IGR J17252-3616) was discovered in 1984 by EXOSAT Galactic plane observations (Warwick et al. 1988). 
Further observations carried out in 1987 by the Ginga satellite showed the presence of pulsations with a $413.9 \pm 0.2 \mathrm{~s}$ period. The X-ray source was found to be highly variable with the $6-21 \mathrm{keV}$ flux decreasing from 2 mcrab to $0.2-0.3$ mcrab over an $8 \mathrm{hr}$ period, with the flux persistent in the $20-60 \mathrm{keV}$ band, but undetectable above $60 \mathrm{keV}$. At maximum flux the luminosity was found to be $5 \times 10^{36} \mathrm{erg} \mathrm{s}^{-1}$ (assuming a distance of $10 \mathrm{kpc}$, Tawara et al. 1989). The orbital period was refined to $9.741 \pm 0.004$ days and the system eclipse duration was determined as $1.7 \pm 0.1$ days (Corbet et al. 2005). Assuming that the donor underfills its Roche lobe and using the eclipse time measurements and the orbital solution from Corbet et al. (2005), implies a donor radius between 21 and $37 R_{\odot}$ with a mass less than $22 M_{\odot}$, and a calculated distance of between 5.3 and $8.7 \mathrm{kpc}$ (Thompson et al. 2007). From this radius and mass range the donor star was proposed to be a supergiant of spectral type B0 I-B5 I. Observations by XMM-Newton allowed a precise determination of the source positon (to within $4^{\prime \prime}$ ) and an IR counterpart 2MASS J17251139-3616575, (with magnitudes $J=14.2, H=11.8$ and $K_{\mathrm{s}}=10.7$ ) was proposed independently by both Zurita Heras et al. (2006) and Negueruela \& Schurch (2007).

\subsection{OAO 1657-415}

OAO 1657-415 was first detected by the Copernicus satellite (Polidan et al. 1978) and later observations with HEAO-1 determined a pulse period of 38.22 s (White \& Pravdo 1979). OAO 1657-415 was subsequently found to exist in an eclipsing $\sim 10.4$ day binary orbit (Chakrabarty et al. 1993). Limitations imposed by the uncertain spectral classification of the mass donor complicate estimates of the distance and hence bolometric X-ray luminosity, although Audley et al. (2006) report a distance of $7.1 \pm 1.3 \mathrm{kpc}$ based on observations of the dust-scattered X-ray halo. Nevertheless, the X-ray properties OAO1657-415 mark it as highly atypical. Its location in the Corbet diagram (Fig. 3) separates it from both SG and Be XRBs, implying that it is transitioning from direct wind fed to disc mediated accretion. This hypothesis is supported by a long term secular spin up of the pulsar on a timescale of $\sim 125 \mathrm{yr}$ (with the superposition of additional brief spin-down and up episodes, Barnstedt et al. 2008); the rapidity of the process arguing for a short lived phase of stellar/binary evolution.

The longstanding question of whether OAO 1657-415 was a high-mass or low-mass system was resolved by the Compton Gamma-Ray Observatory (CGRO) BATSE instrument, deducing from the X-ray pulsar's orbital parameters that the mass donor has a mass of $14-18 M_{\odot}$ with radius $25-32 R_{\odot}$ corresponding to a B0-6 supergiant (Chakrabarty et al. 1993). Subsequent examination by the Chandra X-Ray Observatory determined the precise position of OAO 1657-415 within an error radius of $0.5^{\prime \prime}$. Optical imaging of the field did not detect any stars at the $C$ andra identified position upto a limit of $V>23$. This implied that the companion experienced significant reddening, requiring near infrared observations to reveal the infrared counterpart star coincident with the Chandra position. The IR counterpart 2MASS J17004888-4139214, (with magnitudes $J=14.1$, $H=11.7$ and $\left.K_{\mathrm{s}}=10.4\right)$ was found to have $A_{\mathrm{V}}=20.4 \pm 1.3$ and a distance of $6.4 \pm 1.5 \mathrm{kpc}$ (Chakrabarty et al. 2002).

Near-infrared photometry observations were consistent with the previously reported classification of a B0-B6 supergiant. However, infrared photometry cannot provide an entirely reliable classification, as spectral type and reddening become degenerate on infrared colour-colour diagrams
(Chakrabarty et al. 2002). It is possible to perform reliable spectral classification using infrared spectroscopy, with the recent publication of high $S / N$ and resolution spectral atlases of early $\mathrm{O}$ and B stars (Hanson et al. 2005) and the advent of IR spectroscopy on large telescopes; interest in this technique has increased as more previously inaccessible objects are classified.

\section{Observations and data reduction}

Given the relative faintness of both stars $(K \sim 10.7 \& 10.4$ for EXO 1722-363 and OAO 1657-415 respectively) we utilised VLT/ISAAC to obtain high $\mathrm{S} / \mathrm{N}$ and resolution $(R \sim 3000)$ spectra. The observations were made on 2008 May 17th in the SW MRes mode with a $0.8^{\prime \prime}$ slit. To achieve spectral coverage from 2.0-2.22 $\mu \mathrm{m}$ two exposures were obtained, centred at $2.06 \mu \mathrm{m}$ and $2.15 \mu \mathrm{m}$. Total integration times were 2240s for both EXO 1722-363 and OAO 1657-415, with the resulting data having a count rate below 10000 ADU; therefore no correction for nonlinearity was necessary. Spectra were pipeline reduced and were wavelength calibrated with $\mathrm{OH}$ lines. Finally, telluric correction was made utilising two B5V stars, Hip 085008 and Hip 087805. Unfortunately, due to thin cirrus cloud and highly variable sky conditions, residuals are still present in the vicinity of the He I $2.058 \mu \mathrm{m}$ line in both spectra and the $\mathrm{Br} \gamma$ line for OAO 1657415. Consequently, while we are confident in the identification of these features - noting they are present in the uncorrected target spectra and hence not spuriously introduced by division by the telluric standards - we caution against over interpretation of the detailed line profiles. We present the spectra of both stars in Figs. 1 and 2

\section{Spectral classification of EXO 1722-363}

In Fig. 1 the spectrum of EXO 1772-363 is compared to template $\mathrm{O}$ and $\mathrm{B}$ supergiants from Hanson et al. (2005). All the absorption lines in this spectrum are narrow, indicative of the object being a supergiant. EXO 1722-363 shows the singlet He I $2.058 \mu \mathrm{m}$ line in emission which is highly sensitive to wind and temperature properties, appearing in absorption in mid to late $\mathrm{O}$ stars whilst frequently appearing in emission in early B supergiants (Hanson et al. 2005). The lack of any lines due to the C IV triplet $(2.069,2.078$ and $2.083 \mu \mathrm{m})$ and observed He I $2.058 \mu \mathrm{m}$ emission implies that EXO 1722-362 is not an O type supergiant. The He I $2.058 \mu \mathrm{m}$ line in emission contrasts with that of He I $2.112 \mu \mathrm{m}$ in absorption, this is typical of B0-B2 supergiants (Rahoui \& Chaty 2008). The N III $2.115 \mu \mathrm{m}$ line in emission is a feature common to B0-B1 supergiants. Further evidence pointing to an early B-type classification is the presence of the He I $2.184 \mu \mathrm{m}$ line, seen here in absorption, combined with the lack of an observed He II $2.188 \mu \mathrm{m}$ absorption line, typically absent in spectral types later than 09 (Hanson et al. 2005). The lack of strong $\mathrm{Br} \gamma 2.1655 \mu \mathrm{m}$ emission and the absence of Fe II $2.089 \mu \mathrm{m}$ and $\mathrm{Mg}$ II 2.138 and $2.144 \mu \mathrm{m}$ emission indicates that EXO 1722-363 lacks a strong stellar wind. From a qualitative comparison of spectra from Hanson et al. (2005), we identify EXO 1722-363 as being of spectral type B0-B1 Ia. Although we note that we cannot precisely define the luminosity sub-class for two main reasons, firstly the spectral atlas of Hanson et al. (2005) exhibits a paucity of spectra covering luminosity subclasses lower than BIa, additionally it is difficult to distinguish between luminosity sub-classes in the $K$ band as the spectral features observed are not highly dependant on luminosity.

From recent studies of the physical and wind properties of early B supergiants (Crowther et al. 2006b), we find this spectral 


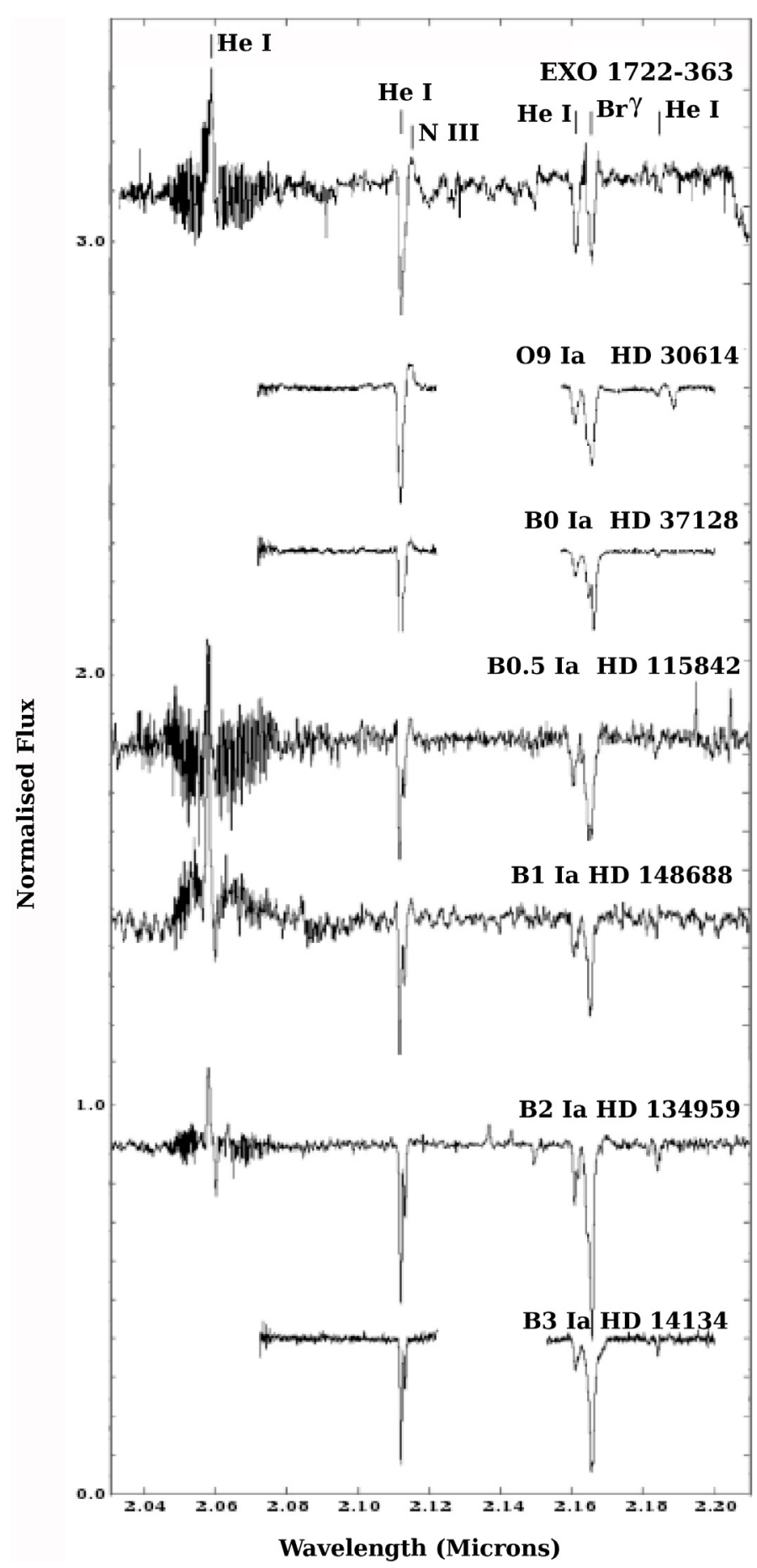

Fig. 1. Comparison of EXO 1722-363 and template 09-B3 Ia spectra from Hanson et al. (2005) in the $K$ band. The fine toothcomb structure at $\lambda=2.06 \mu \mathrm{m}$ is due to imperfect telluric correction.

range has parameters: $22 \mathrm{kK} \leq T_{\text {eff }} \leq 28 \mathrm{kK}, 22_{\odot} \leq R_{*} \leq 36_{\odot}$, $5.35 \leq \log \left(L / L_{\odot}\right) \leq 5.65$. By comparison with evolutionary rotational massive star models (Meynet \& Maeder 2000) we find an initial progenitor mass for EXO 1722-363 in the range $30-40 M_{\odot}$ The mass we have calculated is based on the object's original progenitor mass. However, with B supergiants experiencing mass-loss at rates $M_{\odot}^{-6} \mathrm{yr}^{-1}$ and above (Crowther et al. 2006b), EXO 1722-363 current mass will be significantly reduced from the value we have calculated here.

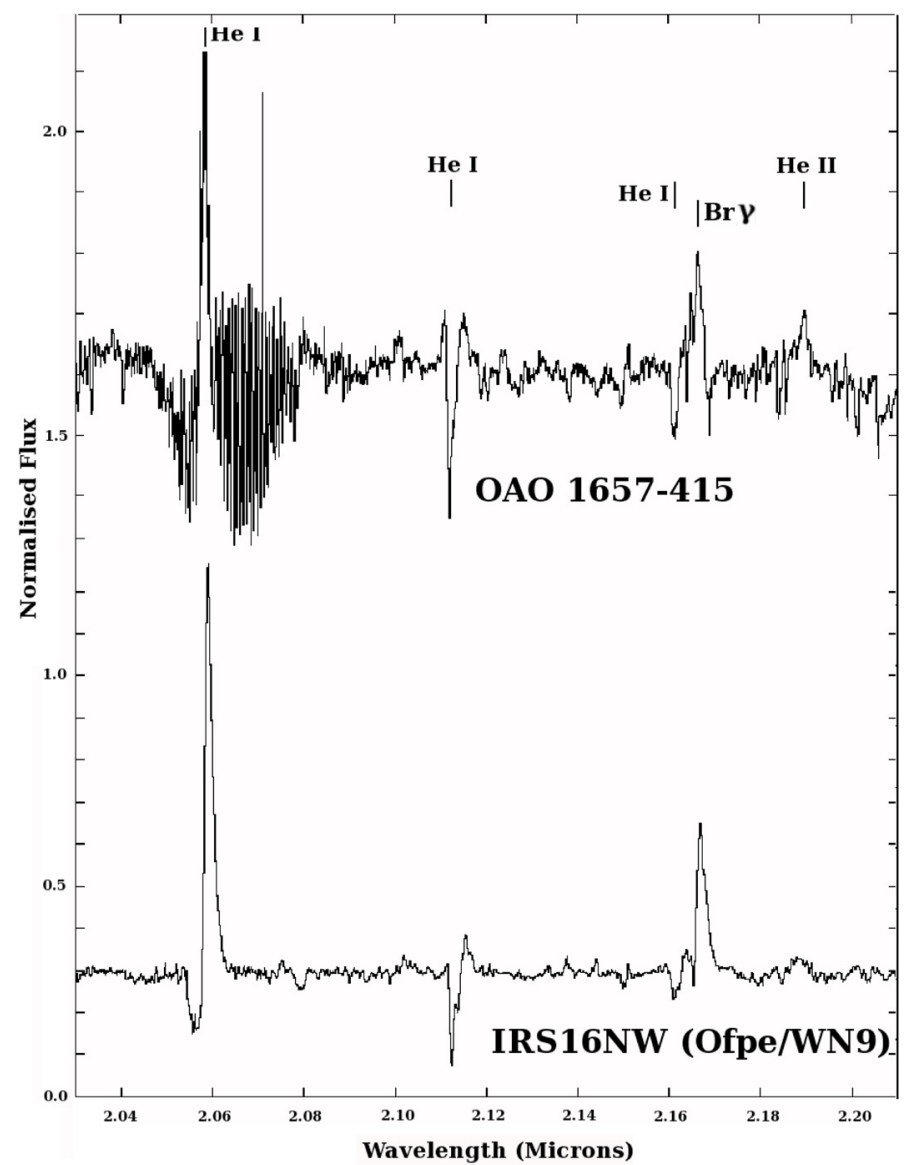

Fig. 2. Comparison of OAO 1657-415 and IRS16NW $K$ band spectra from Martins et al. (2007). The fine toothcomb structure at $\lambda=2.06 \mu \mathrm{m}$ is due to imperfect telluric correction.

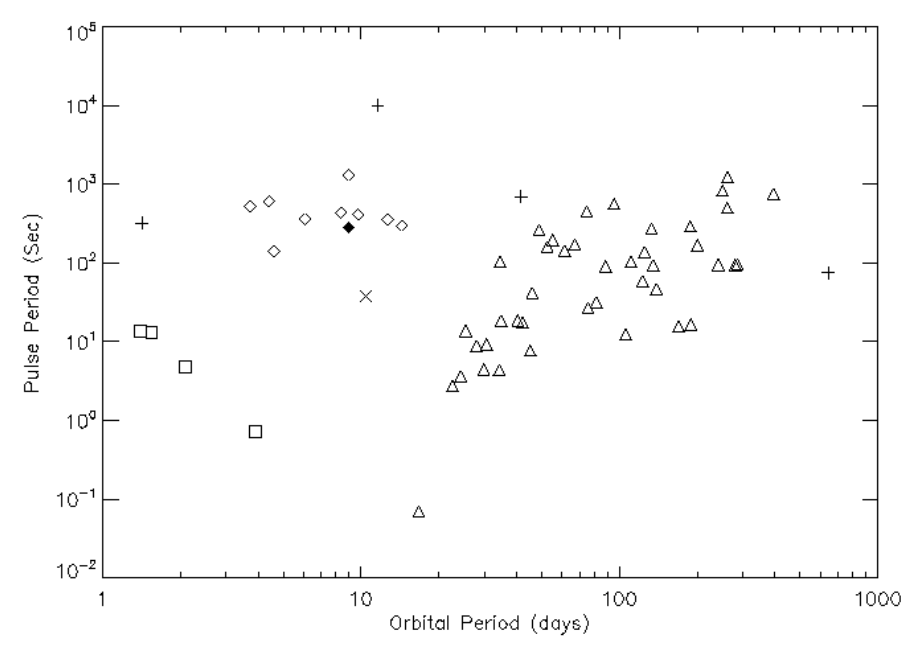

Fig. 3. Corbet diagram marking position of OAO 1657-415 and other HMXBs. OAO 1657-415 is marked by an X, EXO 1722-363 by a filled diamond. SGXRB Roche-Lobe Overflow systems (Squares), Be/X binaries (Triangles), SGXRB Wind-fed systems (Diamonds) and anomalous systems $(+)$.

Following the method for determining spectroscopic distance as detailed in Bibby et al. (2008) and using the parameters shown in Table 1, we determined a distance for EXO $1722-363$ of $8.0_{-2.0}^{+2.5} \mathrm{kpc}$ which is comparable within errors to the distance deduced in Thompson et al. (2007). The quoted 
Table 1. EXO 1722-363 $H$ and $K$ magnitudes, with interstellar extinctions $A_{K_{\mathrm{s}}}$, adopted absolute magnitudes $K_{\mathrm{s}}$ for early B supergiants, distance moduli (DM) from Bibby et al. (2008) with calculated distance.

\begin{tabular}{ccccccccc}
\hline \hline $\begin{array}{c}H \\
(\mathrm{mag})\end{array}$ & $\begin{array}{c}K_{\mathrm{s}} \\
(\mathrm{mag})\end{array}$ & $\begin{array}{c}H-K_{\mathrm{s}} \\
(\mathrm{mag})\end{array}$ & $\begin{array}{c}\left(H-K_{\mathrm{s}}\right)_{0} \\
(\mathrm{mag})\end{array}$ & $\begin{array}{c}E_{\left(H-K_{\mathrm{s}}\right)} \\
(\mathrm{mag})\end{array}$ & $\begin{array}{c}M_{K_{\mathrm{s}}} \\
(\mathrm{mag})\end{array}$ & $\begin{array}{c}A_{K_{\mathrm{s}}} \\
(\mathrm{mag})\end{array}$ & $\begin{array}{c}\text { DM } \\
(\mathrm{mag})\end{array}$ & $\begin{array}{c}\text { Distance } \\
\mathrm{kpc}\end{array}$ \\
\hline 11.81 & 10.67 & 1.14 & -0.08 & 1.22 & $-6.0 \pm 0.5$ & $2.2 \pm 0.4$ & $14.5 \pm 0.6$ & $8.0_{-2.0}^{+2.5}$ \\
\hline
\end{tabular}

uncertainity in the distance estimate principly stems from the uncertainty in absolute magnitude $M_{K_{\mathrm{s}}}$ (where we have adopted the mean values in the range of the B0-B1 I $M_{K_{\mathrm{s}}}$ magnitudes from Bibby et al. 2008) and interstellar extinction $A_{K_{\mathrm{s}}}$. Further work will provide a more accurate and detailed mass estimate from on-going radial velocity studies based upon the $\operatorname{Br} \gamma$ line, this will enable additional refinements to be made to distance and luminosity parameters.

Comparing our calculated distance with model fluxes derived from spectral fits to EXO 1722-363 (Corbet et al. 2005), we found that EXO 1722-363 has an intrinsic X-ray flux variability (in the range $2-60 \mathrm{keV}$ ) such that $F_{\min }=0.78 \times$ $10^{-10} \mathrm{erg} \mathrm{cm}^{-2} \mathrm{~s}^{-1}$ and $F_{\max }=12.2 \times 10^{-10} \mathrm{erg} \mathrm{cm}^{-2} \mathrm{~s}^{-1}$. Using these values combined with the distance range defined above, we derive X-ray luminosities for EXO 1722-363 such that $L_{X_{\min }}=$ $3.4 \times 10^{35} \mathrm{erg} \mathrm{s}^{-1}$ and $L_{X_{\max }}=1.6 \times 10^{37} \mathrm{erg} \mathrm{s}^{-1}$. We find this luminosity range entirely consistent with EXO 1722-363 being the donor within an SGXRB system.

\section{Spectral classification of OAO 1657-415}

The spectrum of the mass donor in OAO1657-415 is presented in Fig. 2, and is dominated by $\mathrm{He} \mathrm{I} 2.058 \mu \mathrm{m}$ and $\mathrm{Br} \gamma$ emission, the former stronger than the latter. He I $2.112 \mu \mathrm{m}$ and the He I complex centred around $2.16 \mu \mathrm{m}$ are seen in aborption, while there is no evidence for high excitation C IV or low excitation Fe II and $\mathrm{Mg}$ II emission features that characterise early-mid $\mathrm{O}$ and midlate B supergiants respectively. Nevertheless we find a poor correspondence with the spectra of B0-6 supergiants (Hanson et al. 1996, 2005) - as suggested for the mass donor by Chakrabarty et al. (2002) on the basis of a combination of photometric and $\mathrm{X}$-ray data.

However, comparison with the spectra of massive transitional objects presented by Morris et al. (1996) is more encouraging. Massive transitional stars are a heterogenous grouping of both cool and hot supergiants, characterised by extreme mass loss rates which act to remove the $\mathrm{H}$ rich mantle of the progenitor as it evolves into a H-depleted Wolf Rayet star. In particular OAO 1657-415 shows pronounced similarities to the hot Ofpe/WNL stars. This is dramatically illustrated by comparison to GC IRS16NW, located within the Galactic Centre cluster (Martins et al. 2007). Unfortunately, such a classification results in interpretational difficulties.

Firstly, such stars demonstrate a wide range of intrinsic luminosities $\left(\log \left(L / L_{\odot}\right) \sim 5.3-6.2\right.$; Clark et al., submitted) and hence progenitor masses $\left(\sim 25-100+M_{\odot}\right.$ respectively). Additionally, such stars also exhibit a known observational degeneracy such that differing combinations of mass loss rate, $\mathrm{H} / \mathrm{He}$ ratio and temperature (and hence luminosity) yield identical $K$ band spectral morphologies (Hillier et al. 1998; Martins et al. 2007). Consequently we may not a priori determine a unique distance to OAO $1657-415$ on the basis of this classification. Adopting the appropriate $\left(J-K_{\mathrm{s}}\right)_{0}$ colour for such stars from Crowther et al. (2006a) and the reddening law of Rieke \& Lebofsky (1985) yields $A_{K_{\mathrm{s}}}=2.37$. Then, assuming a bolometric correction from the non-LTE analysis of GC IRS16NW by Martins et al. (2007) we may infer a minimum and maximum distance for OAO 1657415 by adopting the range of intrinsic luminosities given above. In doing so we find inevitably unconstrictive limits of $4.4 \mathrm{kpc}<$ $d<12 \mathrm{kpc}$. In turn this results in $1.5 \times 10^{36} \mathrm{erg} \mathrm{s}^{-1}<L_{\mathrm{X}}<$ $10^{37} \mathrm{erg} \mathrm{s}^{-1}$, also entirely consistent with observed luminosities of SG HMXBs. As Ofpe/WNL stars typically demonstrate systematically lower wind velocities and higher mass loss rates than normal OB supergiants (see Sect. 4.2), we would expect to observe a higher than average X-ray luminosity than that typically seen in most other SGXRBs. We believe for this source the X-ray luminosity will approach the upper limit of $L_{\mathrm{X}} \sim 10^{37} \mathrm{erg} \mathrm{s}^{-1}$ we have derived, implying that OAO 1657-415 lies at the upper limits of our quoted distance range. Alternatively, adopting the distance derived by Audley et al. (2006) leads to $\log \left(L / L_{\odot}\right) \sim 5.7$. For such a luminosity, comparison to the evolutionary tracks for massive stars from Meynet \& Maeder (2000) imply an initial mass of $\sim 40 M_{\odot}{ }^{1}$. We caution that such a mass estimate does not a priori imply that $\sim 40 M_{\odot}$ stars yield neutron stars post $\mathrm{SNe}$; for example Wellstein \& Langer (1999) propose a scenario for the B Hypergiant+neutron star HMXB GX301-2 in which conservative case A mass transfer from a $26+25 M_{\odot \text {,initial }}$ configuration leads to the current mass of the donor ( $>39 M_{\odot}$ Kaper et al. 2004).

\subsection{Formation and evolution}

With an Ofpe/WNL primary, OAO 1657-415 adds to the growing number of HMXB which have mass donors in a more advanced evolutionary state than the canonical OB supergiants of which EXO 1722-363 is an exemplar. These include the supergiant B[e] systems CI Cam (Clark et al. 1999), IGR J16318-4848 (Filliatre \& Chaty 2004) \& IGR J16358-4726 (Rahoui et al. 2008), the B hypergiant GX301-2 (Kaper et al. 2004) and the Wolf Rayet star mass donors to Cyg X-3 (van Kerkwijk et al. 1992) and IC10 X-1 (Clark \& Crowther 2004). Note that the greater number of Galactic HMXBs with canonical OB supergiants ${ }^{2}$ likely reflects a combination of the relatively short lifetimes of transitional stars and Wolf Rayets with respect to OB supergiants and the fact that lower mass stars $\left(\sim 15-25 M_{\odot}\right)-$ which form viable mass donors for supergiant HMXBs - are not expected to evolve to such advanced evolutionary states.

Given the presence of $\mathrm{Br} \gamma$ emission in the spectrum of OAO 1657-415, we caution that it is less evolved than the H-free Wolf Rayet mass donors of Cyg X-3 and IC10 X-1. Hence it appears unlikely that it represents the post common envelope endpoint of the evolutionary scenario for HMXBs of van den Heuvel \& de Loore (1973); moreso given that such a binary interaction would be expected to yield a very short

\footnotetext{
1 Note that in light of the degeneracy described above, adoption of a higher stellar temperature would result in a systematic reduction in distance estimates, or, for a given distance, an increase in the initial mass of the primary.

2 Which, including Supergiant Fast X-ray transients, currently number in excess of 20 , with a further 10 candidates.
} 
orbital period. Additionally, the non zero eccentricity of the orbit (Chakrabarty et al. 1993) would also argue against a post-SNe common envelope phase. Finally, the Midcourse Souce Experiment (Egan et al. 2001) and the GLIMPSE \& MIPSGAL/Spitzer legacy surveys (Benjamin et al. 2003; Carey et al. 2009) reveal a lack of either point-like or spatially extended excess mid-IR emission that would be indicative of dusty ejecta produced in either a common envelope, Red Supergiant (RSG) or Luminous Blue Variable (LBV phase see Sect. 4.2) Voors et al. (2000); Clark et al. (2003, 2007); Fuchs et al. (2006). Therefore we consider it most likely that the mass donor in OAO 1657-415 has evolved directly into an Ofpe/WNL phase, implying a massive $\left(>40 M_{\odot}\right)$ progenitor - consistent with a distance of $7.1 \pm 1.3 \mathrm{kpc}$ (Audley et al. 2006), rather than through a lower mass $\left(25-40 M_{\odot}\right)$ channel that would require it to be in a post RSG phase (Meynet \& Maeder 2000).

However, in light of the absence of (mid-IR) evidence for significant recent mass loss, this mass estimate appears uncomfortably high given the constraints implied by Chakrabarty et al. (1993) on the basis of the X-ray properties: a current mass for the donor of $14-18 M_{\odot}$ for a $1.4 M_{\odot}$ neutron star, with a maximum of $\sim 25 M_{\odot}$ under restrictive circumstances ${ }^{3}$. We note that the mass of the Ofpe/WNL star within the binary Cyg OB2-5 - which is not expected to have passed through a RSG phase is also surprisingly low $10.5-15.5 M_{\odot}$ (Rauw et al. 1999). Given these observations, one might speculate that binary mass and angular momentum transfer to the Ofpe/WNL stars in both systems would lead to rapid spin up and hence increased mass loss rates (Petrovic et al. 2005), in turn leading to the current, unexpectedly low stellar masses inferred for both stars.

\subsection{X-ray properties}

Finally, we turn to the implications of the Ofpe/WNL classification for the X-ray properties of OAO 1657-415. The anomalous position of OAO 1657-415 within the Corbet diagram (Fig. 3) (Corbet 1986), is then naturally explained in terms of the properties of it's stellar wind. Compared to normal OB supergiants (Crowther et al. 2006b), Ofpe/WNL stars typically demonstrate systematically lower wind velocities and higher mass loss rates (Martins et al. 2007). This combination of wind properties permits a higher accretion rate and hence transfer of angular momentum to the neutron star, in turn leading to a smaller (instantaneous) equilibrium spin period with respect to normal OB supergiants $\left(P_{\text {spin }} \propto \dot{M}^{-3 / 7} v_{\infty}^{12 / 7}\right.$ from Eq. (12) of Waters \& van Kerkwijk 1989, where $P_{\text {spin }}, \dot{M}$ and $v_{\infty}$ are the spin period of the neutron star and the mass loss rate and terminal velocity of the mass donor wind respectively).

Likewise, Ofpe/WNL stars have been proposed to be the hot quisecent state of LBVs - an unstable phase in the post-MS lifetime of massive stars which is characterised by dramatic long term $(\sim \mathrm{yrs})$ changes in both stellar radius and/or mass loss rate (see Humphreys \& Davidson (1994) for a review). If OAO 1657 415 were an incipient/quiescent LBV, then such increases in either mass loss rate or radius - bringing it closer to the Roche Lobe and hence increasing the mass transfer rate - could explain the significant long term ( $\sim$ months) variability in X-ray luminosity that it demonstrates (Kuulkers et al. 2007). Indeed, given both the mass loss rates and radial extent that LBVs have been observed to reach (e.g. $\dot{M} \geq 10^{-4} M_{\odot} \mathrm{yr}^{-1}$ and $R_{*}>100 R_{\odot}$,

3 The requirement that the mass donor is always within its Roche Lobe, with a low inclination angle and neutron star mass.
Clark et al., submitted $)^{4}$ one could anticipate a greatly enhanced mass transfer rate for OAO 1657-415 leading either to an extreme X-ray luminosity or the formation of a common envelope and spiral in/merger of both components in a high mass analogue to the RSG common envelope phase described by van den Heuvel \& de Loore (1973). Given such a potential scenario it is of considerable interest that the B hypergiant HMXB system GX301-2 shows evidence for a pronounced circumstellar envelope of the type that is indicative of the pronounced mass loss associated with the LBV phase (Moon et al. 2007).

\section{Conclusions}

Within this paper we have presented the analysis and results of observations performed at ESO/VLT with the ISAAC spectrometer on the eclipsing high-mass X-ray binaries systems EXO 1722-363 and OAO 1657-415. Using NIR spectrometry we have constrained the previous spectral classification of the mass donor in the EXO 1722-363 system from B0-B5 I to B0-BI Ia and determined its distance to be $8.0_{-2.0}^{+2.5} \mathrm{kpc}$. Examination of the OAO 1657-415 system has allowed us to determine that the donor in this system is more evolved than the typical OB supergiants found in other eclipsing high mass X-ray binaries. We have classified the donor within OAO 1657-415 as type Ofpe/WN9, a transitionary object between OB main sequence stars and hydrogen depleted Wolf-Rayet stars. Due to the large range in luminosity exibited by these types of stars it is difficult to precisely perform distance calculations. Adopting a luminosity range of $\log \left(L / L_{\odot}\right) \sim 5.3-6.2$ we determined a distance range of $4.4 \leq d \leq 12 \mathrm{kpc}$.

The anomolous position of OAO 1657-415 on the Corbet diagram is explained by the more evolved (than typical OB supergiant XRBs) mass donor having a stronger, slower stellar wind, enabling the NS to increase its accretion rate and its rate of spin. This result reinforces our belief that the circumstellar environment from which a HMXB accretes from plays a crucial role in determining its X-ray properties. Results from the INTEGRAL $\gamma$-ray telescope (Walter et al. 2006) have lead to the discovery of two new distinct classes of SG XRB within the past decade. Supergiant Fast X-ray Transients (SFXT) (Negueruela et al. 2006) are believed to stem from periods of high accretion due to wind clumping, and obscured systems such as $\mathrm{sgB}$ [e] stars (Filliatre \& Chaty 2004) have a very high X-ray extinction due to the density of the circumstellar environment. In addition to examining the spin and orbital periods of HMXB systems, it is of vital importance to also consider the properties of the donor's wind. It is increasingly apparent that the X-ray properties of HMXBs are influenced greatly by the circumstellar environment in tandem with the mass-loss properties of the mass donor. We plan to present radial velocity studies of both systems in a future paper, where we shall attempt to determine the masses of the two components in each case.

Acknowledgements. A.B.M. acknowledges support from an STFC studentship. J.S.C. acknowledges support from an RCUK fellowship. This research is partially supported by grants AYA2008-06166-C03-03 and Consolider-GTC CSD2006-00070 from the Spanish Ministerio de Ciencia e Innovación (MICINN). Based on observations carried out at the European Southern Observatory, Chile through programme ID 081.D-0073(A). We also thank the anonymous referee for their useful comments.

\footnotetext{
4 Significantly larger than the orbital separation of OAO 1657-415, projected semimajor axis $a_{\mathrm{x}} \sin i \sim 45 R_{\odot}$, Chakrabarty et al. (1993).
} 


\section{References}

Audley, M. D., Nagase, F., Mitsuda, K., Angelini, L., \& Kelley, R. L. 2006, MNRAS, 367, 1147

Barnstedt, J., Staubert, R., Santangelo, A., et al. 2008, A\&A, 486, 293

Benjamin, R. A., Churchwell, E., Babler, B. L., et al. 2003, PASP, 115, 953

Bibby, J. L., Crowther, P. A., Furness, J. P., \& Clark, J. S. 2008, MNRAS, 386, L23

Carey, S. J., Noriega-Crespo, A., Mizuno, D. R., et al. 2009, PASP, 121, 76

Chakrabarty, D., Grunsfeld, J. M., Prince, T. A., et al. 1993, ApJ, 403, L33

Chakrabarty, D., Wang, Z., Juett, A. M., Lee, J. C., \& Roche, P. 2002, ApJ, 573, 789

Clark, J. S., \& Crowther, P. A. 2004, A\&A, 414, L45

Clark, J. S., Steele, I. A., Fender, R. P., \& Coe, M. J. 1999, A\&A, 348, 888

Clark, J. S., Egan, M. P., Crowther, P. A., et al. 2003, A\&A, 412, 185

Clark, J. S., Barnes, A. D., \& Charles, P. A. 2007, MNRAS, 380, 263

Corbet, R. H. D. 1986, MNRAS, 220, 1047

Corbet, R. H. D., Markwardt, C. B., \& Swank, J. H. 2005, ApJ, 633, 377

Crowther, P. A., Hadfield, L. J., Clark, J. S., Negueruela, I., \& Vacca, W. D. 2006a, MNRAS, 372, 1407

Crowther, P. A., Lennon, D. J., \& Walborn, N. R. 2006b, A\&A, 446, 279

Egan, M. P., Price, S. D., \& Gugliotti, G. M. 2001, in BAAS, 34, 561

Filliatre, P., \& Chaty, S. 2004, ApJ, 616, 469

Fuchs, Y., Koch Miramond, L., \& Ábrahám, P. 2006, A\&A, 445, 1041

Hanson, M. M., Kudritzki, R.-P., Kenworthy, M. A., Puls, J., \& Tokunaga, A. T. 2005, ApJS, 161, 154

Hanson, M. M., Conti, P. S., \& Rieke, M. J. 1996, ApJS, 107, 281

Hillier, D. J., Crowther, P. A., Najarro, F., \& Fullerton, A. W. 1998, A\&A, 340, 483

Humphreys, R. M., \& Davidson, K. 1994, PASP, 106, 1025

Kaper, L., van der Meer, A., \& Tijani, A. H. 2004, in RMxAC, 21, 128
Kuulkers, E., Shaw, S. E., Paizis, A, et al. 2007, A\&A, 466, 595

Liu, Q. Z., van Paradijs, J., \& van den Heuvel, E. P. J. 2006, A\&A, 455, 1165

Martins, F., Genzel, R., Hillier, D. J., et al. 2007, A\&A, 468, 233

Meynet, G., \& Maeder, A. 2000, A\&A, 361, 101

Moon, D.-S., Kaplan, D. L., Reach, W. T., et al. 2007, ApJ, 671, L53

Morris, P. W., Eenens, P. R. J., Hanson, M. M., Conti, P. S., \& Blum, R. D. 1996, ApJ, 470, 597

Negueruela, I., \& Schurch, M. P. E. 2007, A\&A, 461, 631

Negueruela, I., Smith, D. M., Reig, P., Chaty, S., \& Torrejón, J. M. 2006, in The X-ray Universe 2005, ed. A. Wilson, ESA SP-604, 165

Okazaki, A. T., \& Negueruela, I. 2001, A\&A, 377, 161

Petrovic, J., Langer, N., \& van der Hucht, K. A. 2005, A\&A, 435, 1013

Polidan, R. S., Pollard, G. S. G., Sanford, P. W., \& Locke, M. C. 1978, Nature, 275, 296

Rahoui, F., \& Chaty, S. 2008, A\&A, 492, 163

Rahoui, F., Chaty, S., Lagage, P.-O., \& Pantin, E. 2008, A\&A, 484, 801

Rauw, G., Vreux, J.-M., \& Bohannan, B. 1999, ApJ, 517, 416

Rieke, G. H., \& Lebofsky, M. J. 1985, ApJ, 288, 618

Tawara, Y., Yamauchi, S., Awaki, H., et al. 1989, PASJ, 41, 473

Thompson, T. W. J., Tomsick, J. A., in 't Zand, J. J. M., Rothschild, R. E., \& Walter, R. 2007, ApJ, 661, 447

van den Heuvel, E. P. J., \& de Loore, C. 1973, A\&A, 25, 387

van Kerkwijk, M. H., Charles, P. A., Geballe, T. R., et al. 1992, Nature, 355, 703

Voors, R. H. M., Waters, L. B. F. M., de Koter, A., et al. 2000, A\&A, 356, 501

Walter, R., Zurita Heras, J., Bassani, L., et al. 2006, A\&A, 453, 133

Warwick, R. S., Norton, A. J., Turner, M. J. L., Watson, M. G., \& Willingale, R. 1988, MNRAS, 232, 551

Waters, L. B. F. M., \& van Kerkwijk, M. H. 1989, A\&A, 223, 196

Wellstein, S., \& Langer, N. 1999, A\&A, 350, 148

White, N. E., \& Pravdo, S. H. 1979, ApJ, 233, L121

Zurita Heras, J. A., de Cesare, G., Walter, R., et al. 2006, A\&A, 448, 261 